\title{
PHYLLODES TUMOUR: A REVIEW OF AN UNCOMMON BREAST PATHOLOGY AT A SPECIALISED CANCER CENTRE
}

\author{
Samiullah K. Niazi ${ }^{1}$, Raza Sayyed ${ }^{2}$, Muhammad Z. Chaudhry ${ }^{2}$, Amina I. Khan², Huma M. Khan², \\ Shahida Bibi² \\ ${ }^{1}$ Department of Surgery, Dow University of Health Sciences, Karachi, Pakistan, ${ }^{2}$ Department of Surgical Oncology, \\ Shaukat Khanum Memorial Cancer Hospital and Research Centre, Lahore, Pakistan \\ Received: 1 January 2015 / Accepted: 1 April 2016
}

\begin{abstract}
Purpose: Phyllodes tumours are rare breast tumours that comprise almost $1 \%$ of breast tumours. The outcome for these tumours is generally considered better than breast cancers. We review the cases of phyllodes tumour presenting to a specialised cancer centre over a 14-year period.

Materials and Methods: All case records with the diagnosis of phyllodes tumour between 1999 and 2012 were retrieved from the cancer registry. Patient demographics, tumour site, size, axillary lymph node status, whether primary or recurrent, metastatic status, histological type, type of surgery, any complication, margin positivity, postoperative radiation therapy, local or distant recurrence, morality and follow-up duration were recorded. Data were analysed using SPSS.

Results: A total of 77 cases of phyllodes tumour were seen between 1999 and 2012. All patients were female with a mean age of 39.9 years. All patients presented with a breast lump with median duration of 8 months. Almost two-thirds $(65 \%)$ of the patients presented with primary tumour compared to $10 \%$ recurrent tumours and the rest were referred after surgery outside. Median size on histopathology was $5 \mathrm{~cm}$ (IQR 3.5-8.5 cm). Over a median follow-up duration of 31 months (IQR 9-48 months), 69 patients (89.6\%) were alive, while 3 patients (3.9\%) did not survive and 5 patients (6.4\%) were lost to follow-up. Recurrence was seen in $10(13 \%)$ patients with median time to recurrence of 12 months (IQR 7-24). Involved axillary lymph nodes and borderline or malignant histopathology were found to be significantly associated with recurrence $(P=0.04)$, while margin positivity, post-operative radiation therapy and histopathology were not significantly associated with recurrence.
\end{abstract}

Conclusion: Phyllodes tumour is an uncommon breast tumour that is predominantly treated with surgical excision. Although survival with these tumours is better compared to breast cancers, involvement of axillary nodes and borderline or malignant histopathology confer an increased risk of recurrence in these patients.

Key words: Breast cancer, phyllodes tumours, survival

\section{Introduction}

Phyllodes tumour or cystosarcoma phyllodes is a rare breast lesion comprising $<1 \%$ of all breast tumours. ${ }^{[1,2]}$ The usual presentation is a rapidly enlarging mass in a young female, which is often misdiagnosed and treated as fibroadenoma. Quite often, the diagnosis is revealed on histopathology of a presumed fibroadenoma after

Correspondence: Dr. Samiullah K. Niazi, Department of Surgery, Dow University of Health Sciences, Karachi, Pakistan.

Email: drsami80@hotmail.com excision. Pathologically, it varies from benign to borderline or malignant form depending on its size and histological features including mitotic rates. ${ }^{[3]}$ Opinion varies in literature regarding the treatment options, adequate resection margins and role of radiation therapy in prevention of recurrence in this disease. ${ }^{[4-6]}$ There is still controversy about the biological behaviour of borderline and malignant tumours in terms of survival and recurrence.

The most universally acceptable classification system based on pathological features for phyllodes tumours is 
according to the criteria proposed by Azzopardi ${ }^{[3]}$ and Salvadori et al.$^{[5]}$ This classifies these tumours into benign, borderline or malignant based on tumour margins, stromal hypercellularity, stromal overgrowth, tumour necrosis, cellular atypia and mitotic rates per 10 high-power fields.

The incidence of breast cancer in Pakistan is one of the highest in reported literature. ${ }^{[7]}$ With limited resources and a paucity of specialised centres for the treatment of breast cancer, phyllodes tumour is infrequently seen. The purpose of this study was to review clinical presentation, workup, management plan, histopathological features and subsequent follow-up of patients with phyllodes tumour presenting to a specialised breast cancer unit.

\section{Materials and Methods}

A retrospective chart review was performed including all patients with phyllodes tumour presenting to Shaukat Khanum Memorial Cancer Hospital and Research Centre, Lahore. Some of these patients presented with established diagnosis of phyllodes after initial surgery or biopsy of the breast mass elsewhere, while the rest were primarily seen for breast masses and were diagnosed to have phyllodes tumour on the workup of these lesions.

All case records with the diagnosis of phyllodes tumour between 1999 and 2013 were retrieved from the cancer registry. A total of 100 patients were identified who had diagnosis of phyllodes. Of these, 77 cases that underwent surgical treatment at our institution were included in the study. Patient demographics, tumour site, size, axillary lymph node status, primary or recurrent disease, metastatic status, histological type, extent of surgical excision, post-operative complications, margin positivity, post-operative radiation therapy, local or distant recurrence, morality and follow-up duration were recorded. These variables were compared to evaluate any significant difference between patients with benign, borderline and malignant tumours, and also to find any association with the development of recurrent disease.

Kaplan-Meier analysis was conducted to determine if there was any difference in survival between the different pathological groups. Pearson Chi-square test was used to see any relationship of recurrence with size and histopathology. All statistical analyses were carried out using SPSS (Version19 SPSS Inc., Chicago, USA).

\section{Results}

A total of 77 cases of phyllodes tumour were reviewed in this study who presented for treatment between 1999 and 2013. All patients were female with a mean age of 39.9 years (range 16-65 years). Of these patients, 22.1\% were $<30$ years of age, while $15.6 \%$ of these were over 50 years of age. The patient population presented from all areas of Pakistan, but majority (84.4\%) belonged to Punjab. All patients presented with a breast lump with median duration of 8 months. A history of breast lump for $>2$ years was present in $19.7 \%$ of the patients. The distribution of lesions was equal in both breasts (38 right sided vs. 39 left sided). 10 patients (13\%) were unmarried at the time of diagnosis.

Histopathology of patients revealed benign tumours in 31 cases $(40.3 \%)$ and borderline in 22 cases (28.6\%) while remaining 24 cases $(31.2 \%)$ were malignant [Table 1].

Forty-four (57.1\%) patients were treated primarily at out institution as they presented with a palpable breast mass and were worked up for this complaint. Of the remaining patients, 28 (36.4\%) had undergone initial surgery outside, whereas $5(6.5 \%)$ presented with recurrent disease. A total of 42 cases underwent single surgery while others had two or more procedures done for either recurrence or positive margins. Amongst these, 26 patients underwent wide local excision (WLE), four had simple mastectomy and four had sentinel node biopsy with simple mastectomy. Six patients underwent modified radical mastectomy (MRM) as the first procedure, one patient underwent WLE with axillary sampling performed and one had WLE with axillary dissection performed. The reason for axillary nodal sampling or dissection was palpable lymph nodes and large size of the tumours.

Seven patients were initially operated elsewhere and referred to our centre for positive margins. All these patients

Table 1: Histology

\begin{tabular}{l|c}
\hline Histopathology & Frequency (\%) \\
\hline Benign & $31(40.3)$ \\
\hline Borderline & $22(28.6)$ \\
\hline Malignant & $24(31.2)$ \\
\hline Total & $77(100.0)$ \\
\hline
\end{tabular}


had initially undergone lumpectomy. Two had benign lesion and five had borderline lesions. They underwent WLE (two patients; one benign and one borderline) or mastectomy (one benign, large lesion and three borderline lesions). 12 patients were also referred from other hospitals after excision with involved or close margins of malignant phyllodes. Of these six patients underwent reexcision, four had mastectomy and two underwent MRM as they had palpable lymph nodes on presentation.

The mean age for patients with borderline phyllodes was 38 years (range 20-60). Mean duration of symptom at presentation was 17.3 months. Three patients had palpable axillary nodes at presentation, but final histopathology did not reveal any involved lymph nodes. WLE was performed in 18 patients as the first procedure. One patient underwent simple mastectomy, one underwent mastectomy with sentinel lymph node biopsy and two patients underwent MRM. Of all patients with borderline phyllodes, 12 patients primarily presented to our hospital (nine underwent WLE; one each had mastectomy, SLN with mastectomy and MRM). Two patients had reexcision for positive margins. There were four patients who had recurrence of borderline phyllodes in the same breast. Two patients had clear margins after the first surgery and two required reexcision for involved margins. Only one of them had post-operative radiation therapy and subsequently developed recurrence. The other three patients did not receive radiation therapy. One patient had reported mortality in borderline group due to noncancer-related cause. She had clear margins at resection and had no recurrence.

Amongst patients with malignant phyllodes (24), WLE was initial procedure in 15 patients. Five had clear margins and 10 had to undergo repeat surgery due to involved or close margins. Disease recurrence was seen in five patients. Three had lung metastasis, of these two patients had documented mortality and one was lost to followup. There were 18 patients in this group who underwent post-operative radiation therapy. The reasons for offering adjuvant radiation therapy were individualised in each case based on discussion in the multidisciplinary team meeting, but most commonly was size and close margins. One patient amongst these developed diseases recurrence requiring reexcision, while another patient developed lung metastasis.
Median size on histopathology was $5 \mathrm{~cm}$ (IQR 3.5$8.5 \mathrm{~cm}$ ). Of 67 cases, where histopathologic specimen size was mentioned, 31 patients had size between 5 and $10 \mathrm{~cm}$ and 10 patients had $>10 \mathrm{~cm}$ neoplasm.

The median follow-up duration of all patients was 31 months (IQR 9-48 months). Survival was calculated with Kaplan-Meir's chart. Benign phyllodes had 100\% while malignant phyllodes had $91.5 \%$ survival during the follow-up time [Figure 1].

Recurrence was seen in $10(13 \%)$ patients with median time to recurrence 12 months (IQR 7-24). Involved axillary lymph nodes and borderline or malignant histopathology were found to be significantly associated with recurrence $(P=0.04)$. Margin positivity and post-operative radiation therapy were not significantly associated with recurrence.

\section{Discussion}

Phyllodes are a rare tumour of breast. It is reported to account for $<1 \%$ of all female breast tumours. ${ }^{[8]}$ Even in a country like Pakistan where breast cancer incidence is reported to be on the rise, there are few case series reported, suggesting the infrequent occurrence of these tumours. Phyllodes have variable histopathologic characteristics. The histopathological characteristics have mainly been the basis for different histological

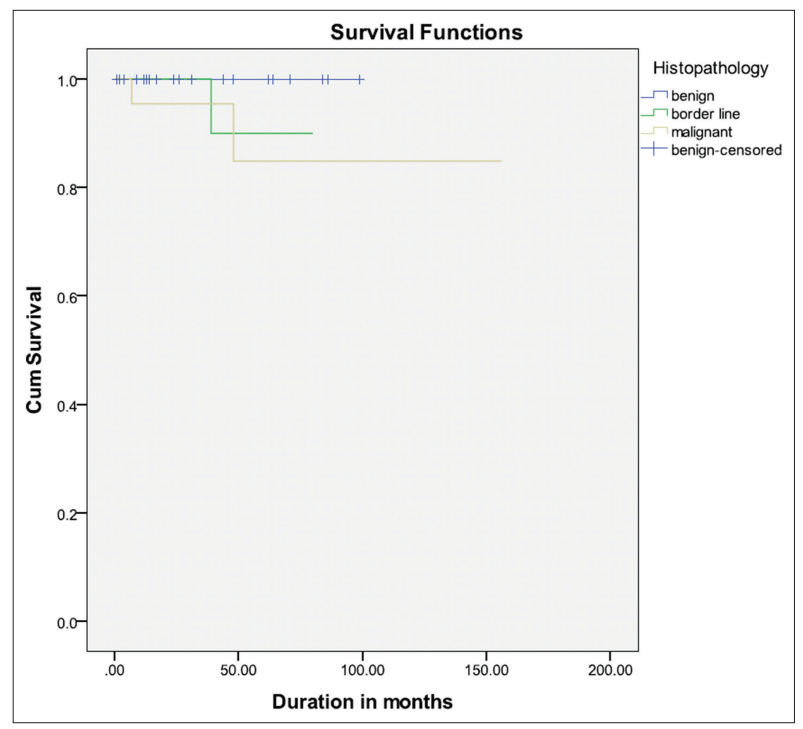

Figure 1: Survival curves for patients with benign, borderline and malignant phyllodes tumour 
classifications, most notably by Treves et al., ${ }^{[9]}$ Pietruszka and Barnes ${ }^{[10]}$ and separately by Azzopardi ${ }^{[3]}$ and Salvadori et al. ${ }^{[5]}$ The World Health Organisation classifies these tumours as low, intermediate and high grade based on histology. ${ }^{[11]}$ Most important histopathological features considered in these classifications are size, number of mitosis per high-power field, invading nature of margins and nuclear features of cells.

Clinical history of patients with phyllodes tumour is also variable; most commonly as slow-growing tumours, with occasional rapid growth resulting in being noticed by the patient. Patients normally do not have any skin involvement or lymph node enlargement. Quite often, these tumours are diagnosed on histopathology of breast lumps removed with clinical diagnosis of fibroadenoma. Unlike fibroadenoma, a significant percentage (10-40\%) of these lesions has malignant potential and tendency for recurrence. ${ }^{[12]}$

We observed that the mean age of our patients was 39.9 years and only $22 \%$ of our patients were $<30$ years. Similar age group was reported by other reviews. ${ }^{[13]}$ A study by Tan et al. ${ }^{[14]}$ reported the mean age of 41.4 years, with $42.3 \%$ of cases younger than 40 years of age. A comparison between Asian and Western population by Chua et al. ${ }^{[15]}$ showed Asian phyllodes patient to be a young female, aged 25-30 years, whereas her Western counterpart is in her 40s. SEER data showed that their patients had a median age of 50 (range 12-96). ${ }^{[16]}$ In comparison, patients at our institution presented mostly with slow-growing tumours with an average duration of the symptoms of 8 months. Almost 20\% of patients had a lump for $>2$ years before any treatment was started. This delay in presentation most likely represents a lack of awareness amongst our population regarding breast complaints.

We observed that histopathology of patients revealed benign in $40 \%$ of cases, while remaining were either borderline $28.6 \%$ or malignant $31 \%$. Tan et al. ${ }^{[14]}$ reported benign cases up to $74.6 \%$, borderline $16.1 \%$ and malignant $9.3 \%$ in their observed group. Another study from Pakistan by Khurshid et al. ${ }^{[17]}$ reported benign $19.1 \%$, borderline $21.9 \%$ and malignant $19.7 \%$ in their study group.

Our patients with benign phyllodes had 100\% while malignant phyllodes have $91.5 \%$ survival during the follow-up period. In comparison, a recent study from Taiwan reported a 5-year disease-free survival was 59\% and the 5-year overall survival to be $81 \%{ }^{[18]}$

In our study, recurrence was seen in $10(13 \%)$ patients with median time to recurrence 12 months. A study from Japan ${ }^{[19]}$ with a median duration of follow-up of 101 months (range 1-273 months) showed local recurrence in six patients $(n=45) ; 5$-, 10- and 15-year cumulative local recurrence-free rates were 88,88 and $84 \%$, respectively, and the cumulative disease-free rates were 85,85 and $81 \%$, respectively. Another study from the United States showed that for patients with non-malignant (benign or indeterminate) and malignant phyllodes, the overall survival was $91 \%$ and $82 \%$, respectively, at 5 years, and $79 \%$ and $42 \%$, respectively, at 10 years. ${ }^{[8]}$

We observed that involved axillary lymph nodes and borderline or malignant histopathology were significantly associated with recurrence $(P=0.04)$, while margin positivity and post-operative radiation therapy were not associated with recurrence in our series. In comparison, a study by Taira et al. from Japan ${ }^{[19]}$ showed the surgical margin $(P=0.0034)$, stromal overgrowth $(P=0.0003)$ and histological classification $(P=0.026)$ as significant predictive factors for local recurrence in univariate analysis. However, in multivariate analysis, the surgical margin was the only independent predictive factor for local recurrence $(P=0.026)$. Various other studies have shown local recurrence rates up to $15-20 \%$ and showed its association with positive excision margins, rather than with tumour grade or size. ${ }^{[20,21]}$

There is no defined treatment strategy for phyllodes tumours and different centres have reported variable outcome with surgery alone, chemotherapy with surgery or radiation with surgery. ${ }^{[22]}$

Some experts consider radiation for borderline tumours excised initially with involved close margins, while other offer radiation therapy to only malignant phyllodes or recurrent benign and borderline tumours. Patients with phyllodes tumour have a favourable prognosis compared to other breast cancers. Mortality is usually associated with metastatic disease or recurrent malignant disease. 


\section{Conclusion}

Phyllodes tumour is an uncommon breast tumour that is predominantly treated with surgical excision. Although survival with these tumours is better compared to breast cancers, involvement of axillary nodes and borderline or malignant histopathology confer an increased risk of recurrence in these patients.

\section{Conflict of Interest}

The authors declare that they have no conflict of interest.

\section{References}

1. Buchanan EB. Cystosarcoma phyllodes and its surgical management. Am Surg 1995;61:350-5.

2. Bernstein L, Deapen D, Ross RK. The descriptive epidemiology of malignant cystosarcoma phyllodes tumors of the breast. Cancer 1993;71:3020-4.

3. Azzopardi JG. Problems in breast pathology. In: Bennington J, editor1. Major Progress in Pathology. Philadelphia, PA: WB Saunders; 1979. p. 346-65.

4. Macdonald OK, Lee CM, Tward JD, et al. Malignant phyllodes tumor of the female breast: Association of primary therapy with cause-specific survival from the surveillance, epidemiology, and end results (SEER) program. Cancer 2006;107:2127-33.

5. Salvadori B, Cusumano F, Del Bo R, et al. Surgical treatment of phyllodes tumors of the breast. Cancer 1989;63:2532-6.

6. Zurrida S, Bartoli C, Galimberti V, et al. Which therapy for unexpected phyllode tumour of the breast? Eur J Cancer 1992;28:654-7.

7. Asif HM, Sultana S, Akhtar N, et al. Prevalence, risk factors and disease knowledge of breast cancer in Pakistan. Asian Pac J Cancer Prev 2014;15:4411-6.

8. Chaney AW, Pollack A, McNeese MD, et al. Primary treatment of cystosarcoma phyllodes of the breast. Cancer 2000;89:1502-11.

9. Treves N, Sunderland DA. Cystosarcoma phyllodes of the breast: A malignant and a benign tumor; a clinicopathological study of seventy-seven cases. Cancer 1951;4:1286-332.

10. Pietruszka M, Barnes L. Cystosarcoma phyllodes: A clinicopathologic analysis of 42 cases. Cancer 1978;41:1974-83.

11. The world health organization histological typing of breast tumors $2^{\text {nd }}$ edition. The world organization. Am J Clin Pathol 1982;78:806-16.

12. Asoglu O, Ugurlu MM, Blanchard K, et al. Risk factors for recurrence and death after primary surgical treatment of malignant phyllodes tumors. Ann Surg Oncol 2004;11:1011-7.

13. Chen WH, Cheng SP, Tzen CY, et al. Surgical treatment of phyllodes tumors of the breast: Retrospective review of 172 cases. J Surg Oncol 2005;91:185-94.

14. Tan PH, Jayabaskar T, Chuah KL, et al. Phyllodes tumors of the breast: The role of pathologic parameters. Am J Clin Pathol 2005; 123:529-40.

15. Chua CL, Thomas A, Ng BK. Cystosarcoma phyllodes asian variations. Aust N Z J Surg 1988;58:301-5.

16. Gullett NP, Rizzo M, Johnstone PA. National surgical patterns of care for primary surgery and axillary staging of phyllodes tumors. Breast J 2009;15:41-4.

17. Khurshid A, Kayani N, Bhurgri Y. Phylloides tumors in adolescent girls and young women in Pakistan. Asian Pac J Cancer Prev 2006; 7:563-6.

18. Lin CC, Chang HW, Lin CY, et al. The clinical features and prognosis of phyllodes tumors: A single institution experience in Taiwan. Int J Clin Oncol 2013;18:614-20.

19. Taira N, Takabatake D, Aogi K, et al. Phyllodes tumor of the breast: Stromal overgrowth and histological classification are useful prognosis-predictive factors for local recurrence in patients with a positive surgical margin. Jpn J Clin Oncol 2007;37:730-6.

20. Mangi AA, Smith BL, Gadd MA, et al. Surgical management of phyllodes tumors. Arch Surg 1999;134:487-92.

21. Barth RJ Jr. Histologic features predict local recurrence after breast conserving therapy of phyllodes tumors. Breast Cancer Res Treat 1999;57:291-5.

22. de Roos WK, Kaye P, Dent DM. Factors leading to local recurrence or death after surgical resection of phyllodes tumours of the breast. Br J Surg 1999;86:396-9. 